\title{
A NEW FRAMEWORK FOR OBJECT WARPING: A SEMI-LAGRANGIAN LEVEL SET APPROACH
}

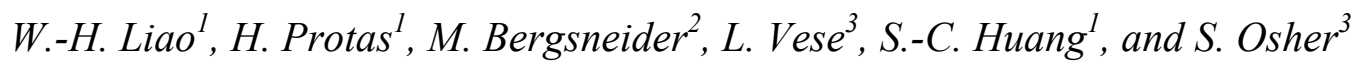 \\ ${ }^{1}$ Department of Biomathematics and Department of Molecular and Medical Pharmacology, UCLA, \\ L.A., CA 90095 \\ ${ }^{2}$ Brain Injury Research Center, UCLA, L.A., CA 90095 \\ ${ }^{3}$ Department of Mathematics, UCLA, L.A., CA 90095 \\ All correspondences should be addressed to the first author at feuillet@ucla.edu
}

\begin{abstract}
In this paper, we present a new framework for object matching between two images. This method could handle multiple pairs of overlapping and non-overlapping shapes, open curves, and landmarks. When implemented in 3-D, the same framework could be used to warp 3-D objects with minimal modification.

Our approach is to use the level set formulation to represent the objects to be matched. Using this representation, the problem becomes an energy minimization problem. Cost functions for warping overlapping, non-overlapping, open curves, and landmarks are proposed. Euler-Lagrange equations are applied and gradient descent is used to solve the corresponding partial differential equations. Moreover, a general framework for linking the level set approach and the infinite dimensional group actions is discussed.
\end{abstract}

\section{INTRODUCTION}

Object warping is a challenging problem which deals with how to find a diffeomorphic transformation that matches one object to the other. It is an important issue in computer vision and pattern recognition as well as many other scientific fields. Recently, image warping has also been an active research area in biomedicine to meet the challenges of representing and comparing different biological structures or images of different modalities. Several strategies of non-rigid warping algorithms have been proposed in the past decade.

Landmark matching and non-rigid dense matching are two important techniques in image warping. Landmark matching involves first identifying userdefined landmarks that need to be matched. By interpolating the discrete matching of the landmarks, one tries to obtain a dense diffeomorphism for the whole image. Dense matching starts by forming a cost function that is minimized when the objects are matched. In order to ensure smooth matching, a regularizing term on the deformation field is added.

In this paper, we will use the terms template and study to denote the images to be matched. Let us denote the template image as $T(x)$ and the study image as $S(x)$ which are images on the spatial domain $\Omega \subset R^{2}$. The problem of image warping is to find a displacement field $u(x)$ at each point $x$ such that a properly defined cost function, which will be denoted by $D(T, S, u)$, between the deformed template and the study is minimized. The displacement field is a vector field such that given any displacement field $u$ the deformed template is given by $T(x-u)$. The term displacement is used because it can be viewed as how a point in the template is moved away from its original location. The most common way to define the measure between the deformed template and the study image is based on the $L^{2}$ norm

$$
D(T, S, u)=\frac{1}{2} \int_{\Omega}|T(x-u)-S(x)|^{2} d x .
$$

Gradient descent of the corresponding Euler-Lagrange equation is often used to minimize this cost function, and an artificial time $t$ has to be introduced in order to solve for the displacement field.

$$
\begin{aligned}
& \frac{\partial u(x, t)}{\partial t}=f(x, u(x, t)) \\
& f(x, u)=\left.[T(x-u)-S(x)] \cdot \nabla T\right|_{x-u} .
\end{aligned}
$$

The function $f(x, u(x, t))$ (up to a sign), which is often called the force field or the body force, describes the derivative of the distance measure with respect to the displacement field $u$.

Unfortunately this problem is known to be ill posed. One way to overcome this difficulty is to add regularization on the underlying deformation. This will be discussed in more details in the next section.

\section{PREVIOUS WORK}

\subsection{Regularizer Based on the Displacement}


How to properly constrain the deformation depends on the nature of the matching. Several models based on regularizing the displacement field $u$ have been proposed. Please refer to the review paper [1] for more details.

\subsection{Diffeomorphisms through Infinite Dimensional Group Actions}

In the past decade, many researchers have tried to establish rigorous theories based on continuum mechanics that ensure diffeomorphic transformation by working on the forward and inverse deformation directly (see [2] and the references therein for a complete description).

In summary, let $g^{-1}(x)=x-u$ which models the deformation field, and $G$ be the group which is formed by all the diffeomorphisms that map $\Omega \in X$ to itself.

A path $g(x, t)=g_{t}(x), g_{t} \in G$ for $\forall t \in[0,1]$, in $G$ is linked to the velocity field $v_{t}$ by the following equation

$$
\frac{\partial}{\partial t} g_{t}(x)=v_{t}\left(g_{t}(x)\right) \text {. }
$$

Once the forward path $g_{t}$ is defined, the inverse path is uniquely determined by $g_{t}^{-1}\left(g_{t}(x)\right)=x$, and is linked to the velocity by $\frac{\partial}{\partial t} g_{t}^{-1}(x)=-D g_{t}^{-1}(x) v_{t}(x)$. Given a path $g(x, t)$ and its associated velocity, we define the energy of the path by

$$
E(g)=\int_{t=0}^{1}\left\|v_{t}(x)\right\|_{L}^{2} d t=\int_{t=0}^{1}\left\langle L v_{t}(x), L v_{t}(x)\right\rangle d t .
$$

Here $L$ is a differential operator. Furthermore, let us define the momentum $p$ by $p(x, t)=p_{t}(x)=L^{\dagger} L v_{t}(x)$, here $L^{\dagger}$ is the adjoint operator of $L$. We then have the following theorem from [2].

\section{Theorem}

Given $g_{0}, g_{1} \in G$, the function $d$ defined as

$$
\begin{aligned}
& d\left(g_{0}, g_{1}\right)= \\
& \left(\inf _{\frac{\partial}{\partial t} g_{t}^{-1}=-D g_{t}^{-1} v_{t}: g_{t=0}=g_{0}, g_{t=1}=g_{1}}^{1}\left\|v_{t=0}(x)\right\|_{L}^{2} d t\right)^{\frac{1}{2}}
\end{aligned}
$$

is a metric on $G$. Moreover, the geodesic satisfies the Euler-Lagrange equation

$$
\frac{d p_{t}}{d t}+\left(v_{t} \cdot \nabla\right) p_{t}+\operatorname{div}\left(v_{t}\right) p_{t}+\sum_{i} \nabla\left(\left(v_{t}\right)_{i}\right)\left(p_{t}\right)_{i}=0 \text {. }
$$

Here $(\bullet)_{i}$ denotes the $i$-th component of a vector.

\section{THEORY}

In this paper, we try to generalize the landmark matching problem by replacing the finite pairs of landmarks to be matched by finite pairs of shapes or curves. We turn to the idea of level set method for ways of representing objects.

To summarize, a shape will be represented by a level set function with the zero level set being the contour of the shape; an open curve will be represented by two level set functions; a landmark will be represented by a level set function, which at any given point takes the value of the distance to this landmark. All level set functions in the template will be denoted by $\varphi$, and those in the study by $\phi$.

\subsection{Distance and Force Field Pairs for Object Matching}

In this section, let us describe briefly the cost functions and the corresponding force fields for matching shapes, open curves and landmarks based on their corresponding level set functions and the setup in 3.2. For derivations, please refer to [3]. Note that all the formulations are in the sense of distribution.

\section{Overlapping shape matching}

$$
\begin{aligned}
& D_{\text {over }}(T, S, t)=\int_{\Omega} H(\phi(x))\left[1-H\left(\varphi\left(g_{t}^{-1}\right)\right)\right] d x \\
& +\int_{\Omega} H\left(\varphi\left(g_{t}^{-1}\right)\right)[1-H(\phi(x))] d x .
\end{aligned}
$$

$$
f_{\text {over }}(x, t)=[1-2 H(\phi(x))] \delta\left(\varphi\left(g_{t}^{-1}\right)\right) \cdot \nabla \varphi\left(g_{t}^{-1}\right) .
$$

\section{Non-overlapping shape matching}

$$
\begin{aligned}
& D_{\text {non }}(T, S, t)=\int_{\Omega}-\varphi\left(g_{t}^{-1}\right) H(\phi(x))\left[1-H\left(\varphi\left(g_{t}^{-1}\right)\right)\right] d x \\
& +\int_{\Omega}-\phi(x) H\left(\varphi\left(g_{t}^{-1}\right)\right)[1-H(\phi(x))] d x . \\
& f_{\text {non }}(x, t)=-\left\{H ( \phi ( x ) ) \left[1-H\left(\varphi\left(g_{t}^{-1}\right)\right)\right.\right. \\
& \left.\left.-\varphi\left(g_{t}^{-1}\right) \delta\left(\varphi\left(g_{t}^{-1}\right)\right)\right]-[1-H(\phi(x))] \phi(x) \delta\left(\varphi\left(g_{t}^{-1}\right)\right)\right\} \\
& \cdot \nabla \varphi\left(g_{t}^{-1}\right) .
\end{aligned}
$$




\section{Open curve matching}

$$
\begin{aligned}
& D(T, S, t)=\int_{\Omega} D_{s}(x) \delta\left(\varphi_{1}\left(g_{t}^{-1}\right)\right)\left|\nabla \varphi_{1}\left(g_{t}^{-1}\right)\right| H\left(\varphi_{2}\left(g_{t}^{-1}\right)\right) d x \\
& +\int_{\Omega} D_{T}\left(g_{t}^{-1}\right) \delta\left(\phi_{1}(x)\right)\left|\nabla \phi_{1}(x)\right| H\left(\phi_{2}(x)\right) d x . \\
& \quad f(x, t)=-\frac{\delta_{1}}{\left|\nabla \varphi_{1}\left(g_{t}^{-1}\right)\right|}\left\{H_{2}<\nabla D_{s}, \nabla \varphi_{1}\left(g_{t}^{-1}\right)>\right. \\
& \left.+D_{s} \delta_{2}<\nabla \varphi_{2}\left(g_{t}^{-1}\right), \nabla \varphi_{1}\left(g_{t}^{-1}\right)>\right\} \nabla \varphi_{1}\left(g_{t}^{-1}\right) \\
& \quad-\delta_{1} D_{s} H_{2} \operatorname{div}\left(\frac{\nabla \varphi_{1}\left(g_{t}^{-1}\right)}{\left|\nabla \varphi_{1}\left(g_{t}^{-1}\right)\right|}\right) \mid \varphi_{1}\left(g_{t}^{-1}\right) \\
& \quad+D_{s} \delta_{1} \delta_{2}\left|\nabla \varphi_{1}\left(g_{t}^{-1}\right)\right| \nabla \varphi_{2}\left(g_{t}^{-1}\right) \\
& +\delta\left(\phi_{1}\right)\left|\nabla \phi_{1}\right| H\left(\phi_{2}\right) \nabla D_{T}\left(g_{t}^{-1}\right) .
\end{aligned}
$$

Here $D_{T}$ and $D_{S}$ are the distance functions to the curves in the template and study respectively, and the following notations are used

$$
\delta_{1}=\delta\left(\varphi_{1}\left(g_{t}^{-1}\right)\right) ; H_{2}=H\left(\varphi_{2}\left(g_{t}^{-1}\right)\right) ; \delta_{2}=\delta\left(\varphi_{2}\left(g_{t}^{-1}\right)\right) \text {. }
$$

\section{Landmark matching}

$$
\begin{aligned}
& D_{\text {landmark }}(T, S, t) \\
& =\int_{\Omega} D_{s} \delta\left(\varphi_{1}\left(g_{t}^{-1}\right)\right) \delta\left(\varphi_{2}\left(g_{t}^{-1}\right)\right)\left|\nabla \varphi_{1}\left(g_{t}^{-1}\right) \times \nabla \varphi_{2}\left(g_{t}^{-1}\right)\right| d x \\
& +\int_{\Omega} D_{T}\left(g_{t}^{-1}\right) \delta\left(\varphi_{1}\right) \delta\left(\varphi_{2}\right)\left|\nabla \varphi_{1} \times \nabla \varphi_{2}\right| d x \\
& f_{\text {landmark }}(x, t) \\
& =-\operatorname{div}\left(\frac{P_{\nabla \varphi_{2}\left(g_{t}^{-1}\right)} \nabla \varphi_{1}\left(g_{t}^{-1}\right)}{\left|P_{\nabla \varphi_{2}\left(g_{t}^{-1}\right)} \nabla \varphi_{1}\left(g_{t}^{-1}\right)\right|}\left|\nabla \varphi_{2}\left(g_{t}^{-1}\right)\right| D_{s}\right) \delta_{1} \delta_{2} \nabla \varphi_{1}\left(g_{t}^{-1}\right) \\
& -\operatorname{div}\left(\frac{P_{\nabla \varphi_{1}\left(g_{t}^{-1}\right)} \nabla \varphi_{2}\left(g_{t}^{-1}\right)}{\left|P_{\nabla \varphi_{1}\left(g_{t}^{-1}\right)} \nabla \varphi_{2}\left(g_{t}^{-1}\right)\right|}\left|\nabla \varphi_{1}\left(g_{t}^{-1}\right)\right| D_{s}\right) \delta_{1} \delta_{2} \nabla \varphi_{2}\left(g_{t}^{-1}\right) \\
& +\delta\left(\phi_{1}\right) \delta\left(\phi_{2}\right)\left|\nabla \phi_{1} \times \nabla \phi_{2}\right| \nabla D_{T}\left(g_{t}^{-1}\right)
\end{aligned}
$$

with

$$
P_{v}=I-\frac{v \otimes v}{|v|^{2}}
$$

\subsection{Incorporating the Distance Measure into Infinite Dimensional Group Actions}

The cost function and force field pairs could be easily integrated into the framework of 2.2. For instance, we have the following theorems, which are extensions of the theorems in [2].

\section{Theorem}

The path that minimizes the following inexact matching problem

$$
\inf _{\frac{\partial}{\partial t} g_{t}^{-1}=-D g_{t}^{-1} v_{t}, g_{t=0}=i d} \int_{t=0}^{1}\left\|v_{t}(x)\right\|_{L}^{2} d t+D(T, S, t=1)
$$

satisfies equation (6) and the boundary condition at $\mathrm{t}=1$

$$
p(x, 1)-\left[f(x, 1)^{T} D g_{t=1}^{-1}\right]^{T}=0 .
$$

\section{Theorem}

The path that minimizes the following space-time matching problem

$$
\inf _{\frac{\partial}{\partial t} g_{t}^{-1}=-D g_{t}^{-1} v_{t}, g_{t=0}=i d} \int_{t=0}^{1}\left(\left\|v_{t}(x)\right\|_{L}^{2}+D(T, S, t)\right) d t
$$

satisfies the Euler-Lagrange equation

$$
\begin{aligned}
& \frac{d p_{t}}{d t}+\left(v_{t} \cdot \nabla\right) p_{t}+\operatorname{div}\left(v_{t}\right) p_{t}+\sum_{i} \nabla\left(\left(v_{t}\right)_{i}\right)\left(p_{t}\right)_{i} \\
& +\left[f(x, t)^{T} D g_{t}^{-1}\right]^{T}=0 .
\end{aligned}
$$

Here $D(T, S, t)$ and $f$ are the cost function and force field pairs defined in 3.1 .

\section{IMPLEMENTATION}

For simplicity, we will choose the Horn and Schunck functional for regularizing the displacement. The advantage of this regularizer is that it is in the form of inhomogeneous heat equations and thus fast solver exists via the additive operator splitting method. Please refer to [3] for implementation details.

\section{RESULTS}

In this paper, all the images are of size 128 by 128 pixels. The grid size is 0.1 . The numerical approximations for calculating the Heaviside function and the delta function could be found in [4].

Figures 1 and 3 are two matching problems (matching non-overlapping ring shapes in figure 1, and arcs in figure 3) where (a) being the template and (b) the study. Figures 2 and 4 are the final deformed templates with underlying grid deformations. Figure 5 is the transformation that deforms landmark pair $(40,50)$ and 
$(80,70)$ to $(50,40)$ and $(70,80)$ respectively. For more details, please refer to [3].

\section{ACKNOWLEDGEMENT}

This paper was supported by NIH grant NS30308 and P20MH65166 and DoE Grant DE-FC0387ER60615.

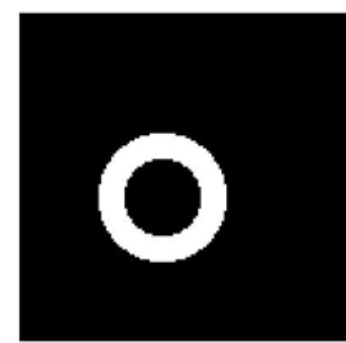

(a)

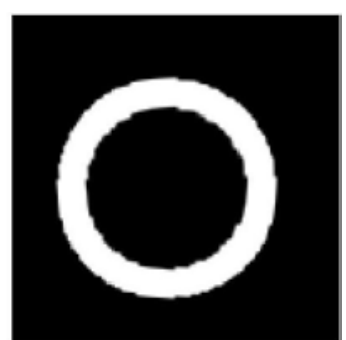

Figure 1. Illustration of a matching problem with two nonoverlapping rings in the template (a) and the study (b).

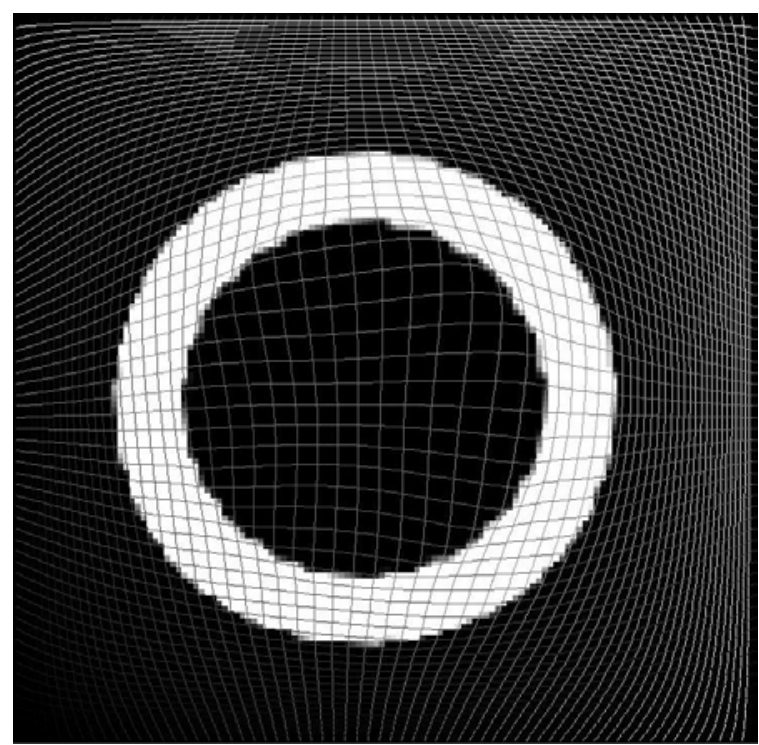

Figure 2. The deformation field obtained by the proposed method for matching figure 1 (a) to 1 (b).
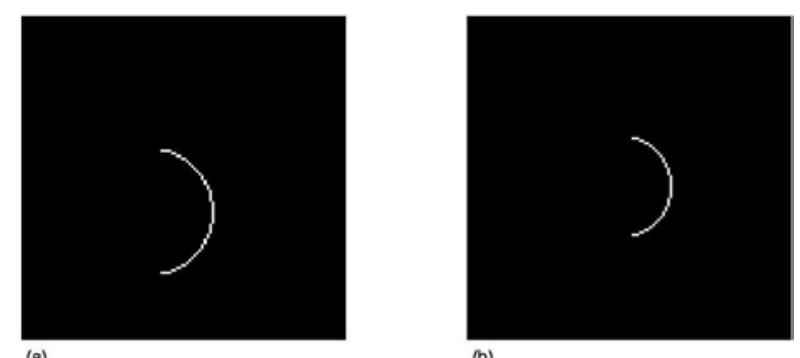

Figure 3. Illustration of a matching problem with two arcs in the template (a) and the study (b).

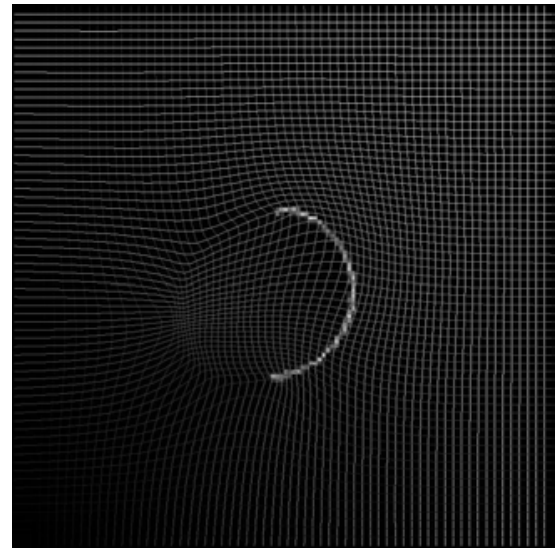

Figure 4. The deformation field obtained by the proposed method for matching figure 3 (a) to 3 (b).

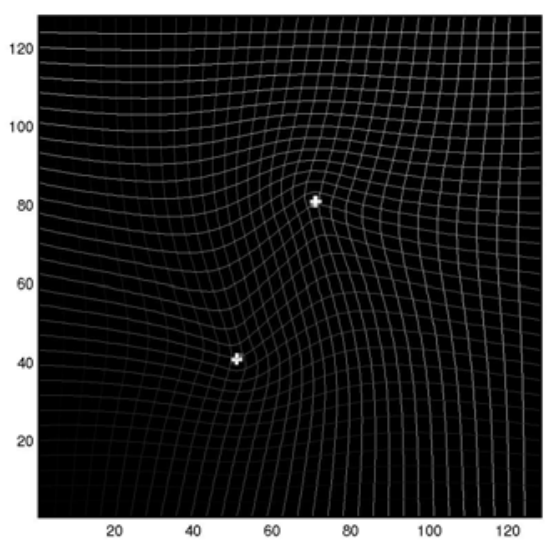

Figure 5. The deformation field obtained by matching starting $(40,50)$ to $(50,40)$, and $(80,70)$ to $(70,80)$.

\section{REFERENCES}

[1] J. Weickert and C. Schnorr, "A theoretical framework for convex regularizers in PDE-based computation of image motion," International Journal of Computer Vision, vol. 45, pp. 245-264, 2001

[2] M. I. Miller, A. Trouve, and L. Younes, "On the metrics and Euler-Lagrange equations of computational anatomy," Annual Review of Biomedical Engineering, vol. 4, pp. 375-405, 2002.

[3] W. H. Liao, A. Khuu, M. Bergsneider, L. A. Vese, S. C. Huang, and S. Osher, "From Landmark Matching to Shape and Open Curve Matching: A Level Set Approach," in UCLA CAM report, 2002.

[4] T. F. Chan and L. A. Vese, "Active contours without edges," Ieee Transactions on Image Processing, vol. 10, pp. 266-277, 2001. 